\title{
A CLASS OF MARKOV CHAINS WITH NO SPECTRAL GAP
}

\author{
YEVGENIY KOVCHEGOV AND NICHOLAS MICHALOWSKI
}

(Communicated by Edward C. Waymire)

\begin{abstract}
In this paper we extend the results of the research started by the first author in which Karlin-McGregor diagonalization of certain reversible Markov chains over countably infinite general state spaces by orthogonal polynomials was used to estimate the rate of convergence to a stationary distribution.

We use a method of Koornwinder to generate a large and interesting family of random walks which exhibits a lack of spectral gap, and a polynomial rate of convergence to the stationary distribution. For the Chebyshev type subfamily of Markov chains, we use asymptotic techniques to obtain an upper bound of order $O\left(\frac{\log t}{\sqrt{t}}\right)$ and a lower bound of order $O\left(\frac{1}{\sqrt{t}}\right)$ on the distance to the stationary distribution regardless of the initial state. Due to the lack of a spectral gap, these results lie outside the scope of geometric ergodicity theory.
\end{abstract}

\section{INTRODUCTION}

Let $P=(p(i, j))_{i, j \in \Omega}$ be a reversible Markov chain over a sample space $\Omega$; that is, it must satisfy the following detailed balance conditions:

$$
\pi_{i} p(i, j)=\pi_{j} p(j, i) \quad \forall i, j \in \Omega,
$$

where $\pi$ is a non-trivial non-negative function over $\Omega$. If $P$ admits a unique stationary distribution $\nu$, then $\frac{1}{\sum_{i \in \Omega} \pi_{i}} \pi=\nu$.

It can be shown that the reversible $P$ is a self-adjoint operator in $\ell^{2}(\pi)$, the space generated by the following inner product induced by $\pi$ :

$$
\langle f, g\rangle_{\pi}=\sum_{i \in \Omega} f(i) g(i) \pi_{i}
$$

If $P$ is a tridiagonal operator (i.e. a nearest-neighbor random walk) on $\Omega=\{0,1,2$, $\ldots\}$, then it must have a simple spectrum and is diagonalizable via orthogonal polynomials, as was studied in the 50's by Karlin and McGregor; see [3], 10], and [2]. There, the extended eigenfunctions $Q_{j}(\lambda)$ satisfying $Q_{0} \equiv 1$ and

$$
P\left(\begin{array}{c}
Q_{0}(\lambda) \\
Q_{1}(\lambda) \\
Q_{2}(\lambda) \\
\vdots
\end{array}\right)=\lambda\left(\begin{array}{c}
Q_{0}(\lambda) \\
Q_{1}(\lambda) \\
Q_{2}(\lambda) \\
\vdots
\end{array}\right)
$$

Received by the editors September 26, 2011 and, in revised form, September 28, 2011 and February 9, 2012.

2010 Mathematics Subject Classification. Primary 33C45, 60G05.

(C) 2013 American Mathematical Society Reverts to public domain 28 years from publication 
are orthogonal polynomials with respect to a probability measure $\psi$. If we let $p_{t}(i, j)$ denote the entries of the operator $P^{t}$ that represent $t$ step transition probabilities from state $i$ to state $j$, then

$$
p_{t}(i, j)=\pi_{j} \int_{-1}^{1} \lambda^{t} Q_{i}(\lambda) Q_{j}(\lambda) d \psi(\lambda) \forall i, j \in \Omega,
$$

where $\pi_{j}$ with $\pi_{0}=1$ is the reversibility measure of $P$.

We will use the following distance to measure the deviation from the stationary distribution on a scale from zero to one.

Definition 1. If $\mu$ and $\nu$ are two probability distributions over a sample space $\Omega$, then the total variation distance is

$$
\|\nu-\mu\|_{T V}=\frac{1}{2} \sum_{x \in \Omega}|\nu(x)-\mu(x)|=\sup _{A \subset \Omega}|\nu(A)-\mu(A)| .
$$

Let $\rho=\sum_{k=0}^{\infty} \pi_{k}$. Observe that $\rho<\infty$ if and only if the random walk $P$ is positive recurrent. Recall that $\nu=\frac{1}{\rho} \pi$ is the stationary probability distribution. If in addition to being positive recurrent, the aperiodic nearest neighbor Markov chain originates at site $j$, then the total variation distance between the distribution $\mu_{t}=\mu_{0} P^{t}$ and $\nu$ is given by

$$
\left\|\nu-\mu_{t}\right\|_{T V}=\frac{1}{2} \sum_{n \in \Omega} \pi_{n}\left|\int_{(-1,1)} \lambda^{t} Q_{j}(\lambda) Q_{n}(\lambda) d \psi(\lambda)\right|,
$$

as measure $\psi$ contains a point mass of weight $\frac{1}{\rho}$ at 1 . See [6].

The rates of convergence are quantified via mixing times, which for an infinite state space with a unique stationary distribution are defined as follows. Here the notion of a mixing time depends on the state of origination $j$ of the Markov chain. See [7.

Definition 2. Suppose $P$ is a Markov chain with a stationary probability distribution $\nu$ that commences at $X_{0}=j$. Given an $\epsilon>0$, the mixing time $t_{m i x}(\epsilon)$ is defined as

$$
t_{\text {mix }}(\epsilon)=\min \left\{t:\left\|\nu-\mu_{t}\right\|_{T V} \leq \epsilon\right\} .
$$

In the case of a nearest-neighbor process on $\Omega=\{0,1,2, \ldots\}$ commencing at $j$, the corresponding mixing time has the following simple expression in orthogonal polynomials:

$$
t_{\text {mix }}(\epsilon)=\min \left\{t: \sum_{n} \pi_{n}\left|\int_{(-1,1)} \lambda^{t} Q_{j}(\lambda) Q_{n}(\lambda) d \psi(\lambda)\right| \leq 2 \epsilon\right\} .
$$

Investigations into the use of orthogonal polynomial techniques (see [3], 10]) in the estimation of mixing times and distance to the stationary distribution has been carried out in [7] for certain classes of random walks. In this paper we consider the problem from the other direction. Namely, given a large class of orthogonal polynomials we outline how to find the corresponding random walk and estimate the rate for the distance to the stationary distribution.

More specifically, beginning with the Jacobi polynomials whose weight function lies in $(-1,1)$, we use Koornwinder's techniques [5] to attach a point mass at 1. For the class of Jacobi type polynomials $Q_{n}$ thus obtained, the three term recurrence relationship is understood [4. The tridiagonal operator corresponding to 
these polynomials is not a Markov chain; however, the operator can be deformed to become one. The corresponding changes in the polynomials are easy to trace. This gives a four parameter family of nearest neighbor Markov chains whose distance to the stationary distribution decays in a non-geometric way. In principle the asymptotic analysis presented in this paper can be applied to the entire four parameter family. We outline how this proceeds for a Chebyshev type subfamily consisting of taking $\alpha=\beta=-1 / 2$ in the Koornwinder class.

We would like to point out the important results of V. B. Uvarov 11 on the transformation of orthogonal polynomial systems by attaching point masses to the orthogonality measure, predating the Koornwinder results by fifteen years. The results of $\mathrm{V}$. B. Uvarov can potentially be used in order to significantly extend the scope of convergence rate problems covered in this current manuscript.

The paper is organized as follows. In Section 2 we discuss constructing positive recurrent Markov chains from the Jacobi family of orthogonal polynomials adjusted by using Koornwinder's techniques to place a point mass at $x=1$. Next, we derive an asymptotic upper bound on the total variation distance to the stationary distribution in the case of general $\alpha>-1$ and $\beta>-1$ in Section 3. Our main result, Theorem 2 is presented in Section 4, There, for the case of Chebyshev type polynomials corresponding to $\alpha=\beta=-1 / 2$, we produce both asymptotic lower and upper bounds for the total variation distance. Finally, in Section 5 we compare our main result to related results obtained by other techniques.

\section{FROM ORTHOGONAL POLYNOMIALS TO RANDOM WALKS VIA KOORNWINDER}

T. Koornwinder [5] provides a method for finding the orthogonal polynomials whose weight distribution is obtained from the standard Jacobi weight functions $C_{\alpha, \beta}(1-x)^{\alpha}(1+x)^{\beta}$ by attaching weighted point masses at -1 and 1 . A spectral measure corresponding to a Markov chain contains a point mass at -1 if and only if the Markov chain is periodic. A spectral measure for an aperiodic Markov chain contains a point mass at 1 if and only if it is positive recurrent. Thus in order to create a class of positive recurrent aperiodic Markov chains with a Koornwinder type orthogonal polynomial diagonalization we will only need to attach a point mass at 1 and no point mass at -1 .

Let $N \geq 0$ and let $\alpha, \beta>-1$. For $n=0,1,2, \ldots$ define

$$
P_{n}^{\alpha, \beta, N}(x)=\left(\frac{(\alpha+\beta+2)_{n-1}}{n !}\right) A_{n}\left[-N(1+x) \frac{d}{d x}+B_{n}\right] P_{n}^{\alpha, \beta}(x),
$$

where

$$
\begin{gathered}
A_{n}=\frac{(\alpha+1)_{n}}{(\beta+1)_{n}} \\
B_{n}=\frac{(\beta+1)_{n} n !}{(\alpha+1)_{n}(\alpha+\beta+2)_{n-1}}+\frac{n(n+\alpha+\beta+1) N}{(\alpha+1)}
\end{gathered}
$$

$P_{n}^{\alpha, \beta}$ is the standard Jacobi polynomials of degree $n$ and order $(\alpha, \beta)$, and $(x)_{n}=$ $x(x+1) \cdots(x+n-1)$. These polynomials form a system of orthogonal polynomials with respect to the probability measure $d \psi(x)=\frac{C_{\alpha, \beta}(1-x)^{\alpha}(1+x)^{\beta} d x+N \delta_{1}(x)}{N+1}$, where $C_{\alpha, \beta}=\frac{1}{\mathcal{B}(\alpha+1, \beta+1)}, \mathcal{B}(\cdot, \cdot)$ is the beta function, and $\delta_{1}(x)$ denotes a unit point mass measure at $x=1$. See T. Koornwinder [5]. Direct calculation shows that 
$P_{n}^{\alpha, \beta, N}(1)=\frac{(\alpha+1)_{n}}{n !}$, and so we normalize $Q_{n}(x)=n ! P_{n}^{\alpha, \beta, N}(x) /(\alpha+1)_{n}$, which is the orthogonal set of polynomials with respect to $d \psi$ satisfying $Q_{n}(1)=1$.

As we mentioned earlier, the tridiagonal operator $H$ corresponding to the recurrence relation of the orthogonal polynomials may not be a Markov chain operator. Let $p_{i}, r_{i}$ and $q_{i}$ denote the coefficients in the tridiagonal recursion

$$
p_{i} Q_{i+1}(x)+r_{i} Q_{i}(x)+q_{i} Q_{i-1}(x)=x Q_{i}(x),
$$

for $i=0,1,2, \ldots$, where we let $Q_{-1} \equiv 0$ as always.

Notice that because the polynomials are normalized so that $Q_{i}(1)=1$, it follows immediately that $p_{i}+r_{i}+q_{i}=1$. However, some of the coefficients $p_{i}, r_{i}$, or $q_{i}$ may turn out to be negative, in which case the rows of the tridiagonal operator $A$ would add up to one but will not necessarily consist of all non-negative entries.

In the case when all the negative entries are located on the main diagonal, this may be overcome by considering the operator $\frac{1}{\lambda+1}(H+\lambda I)$. For $\lambda \geq-\inf _{i} r_{i}$ this ensures all entries in the matrix $\frac{1}{\lambda+1}(H+\lambda I)$ are non-negative and hence can be thought of as transition probabilities. More generally, if a polynomial $p(\cdot)$ with coefficients adding up to one is found to satisfy $p(H) \geq 0$ coordinate-wise, then such $p(H)$ would be a Markov chain.

\section{An ASYMPTOTIC UPPER BOUND FOR JACOBI TYPE POLYNOMIALS}

In this section we derive asymptotic estimates for the distance to the stationary distribution when our operator given by $P_{\lambda}=\frac{1}{\lambda+1}(H+\lambda I)$ is a Markov chain. In this case the Karlin-McGregor orthogonal polynomials for $P_{\lambda}$ are $Q_{j}((1+\lambda) x-\lambda)$ and the orthogonality probability measure is $\frac{1}{1+\lambda} d \psi((1+\lambda) x-\lambda)$ over $\left(\frac{\lambda-1}{\lambda+1}, 1\right]$, where the $Q_{j}$ are the Jacobi type polynomials introduced by Koornwinder in the previous section.

Of course the new operator $P_{\lambda}$ is again tridiagonal. For the $n$-th row of $P_{\lambda}$, let us denote the $(n-1)$-st, $n$-th, and $(n+1)$-st entries by $q_{n}^{\lambda}, r_{n}^{\lambda}$, and $p_{n}^{\lambda}$ respectively. Here the entries of $P_{\lambda}$ can be expressed via the entries of $H$ as follows:

$$
p_{n}^{\lambda}=\frac{p_{n}}{1+\lambda}, \quad r_{n}^{\lambda}=\frac{r_{n}+\lambda}{1+\lambda}, \quad \text { and } \quad q_{n}^{\lambda}=\frac{q_{n}}{1+\lambda} .
$$

Clearly we still have that $p_{n}^{\lambda}+r_{n}^{\lambda}+q_{n}^{\lambda}=1$.

With the probabilities in hand we now compute the corresponding reversibility function $\pi_{n}^{\lambda}$ of $P_{\lambda}$ which is equal to the corresponding function of $H$ defined as $\pi_{n}=\frac{p_{0} \cdots p_{n-1}}{q_{1} \cdots q_{n}}$. Here $\pi_{0}^{\lambda}=1=\pi_{0}$ and $\pi_{n}^{\lambda}=\frac{p_{0}^{\lambda} \cdots p_{n-1}^{\lambda}}{q_{1}^{\lambda} \cdots q_{n}^{\lambda}}=\frac{p_{0} \cdots p_{n-1}}{q_{1} \cdots q_{n}}=\pi_{n}$.

Changing variables in (1.1) yields

$$
\left\|\nu-\mu_{t}\right\|_{T V}=\frac{1}{2} \sum_{n=0}^{\infty} \pi_{n}\left|\int_{(-1,1)}\left(\frac{x}{1+\lambda}+\frac{\lambda}{1+\lambda}\right)^{t} Q_{j}(x) Q_{n}(x) d \psi(x)\right| .
$$

Lemma 1. Consider the case when $p_{n}>0$ and $q_{n}>0$ for all $n \geq 0$, and $\infty>\lambda \geq-\inf _{i} r_{i}$. Then, for the Jacobi type polynomials $Q_{j}$, the distance to the 
stationary distribution satisfies the bound

$$
\left\|\nu-\mu_{t}\right\|_{T V} \leq \frac{C_{\alpha, \beta, \lambda}\left\|Q_{j}\right\|_{\infty}}{(t+1)^{1+\alpha}} \sum_{n=0}^{t+j} \pi_{n}\left\|Q_{n}\right\|_{\infty}+\frac{1}{2} \sum_{n=j+t+1}^{\infty} \pi_{n}
$$

for a certain constant $C_{\alpha, \beta, \lambda}$.

Proof. For $n>j+t$, it follows from the orthogonality of the polynomials and our normalization $Q_{i}(1)=1$ that

$$
\int_{(-1,1)}\left(\frac{x}{1+\lambda}+\frac{\lambda}{1+\lambda}\right)^{t} Q_{j}(x) Q_{n}(x) d \psi(x)=1 .
$$

It is then easy to see that $\|\nu-\mu\|_{T V} \leq I+I I+\frac{1}{2} \sum_{n=j+t+1}^{\infty} \pi_{n}$, where

$$
\begin{gathered}
I=\frac{1}{2} \sum_{n=0}^{j+t} \pi_{n} \int_{(-1,0)}\left|\left(\frac{x+\lambda}{1+\lambda}\right)^{t} Q_{j}(x) Q_{n}(x)\right|(1-x)^{\alpha}(1+x)^{\beta} d x \\
\text { and } \quad I I=\frac{1}{2} \sum_{n=0}^{j+t} \pi_{n} \int_{(0,1)}\left(\frac{x+\lambda}{1+\lambda}\right)^{t}\left|Q_{j}(x) Q_{n}(x)\right|(1-x)^{\alpha}(1+x)^{\beta} d x .
\end{gathered}
$$

To estimate $I$ notice that $\left|\frac{x+\lambda}{1+\lambda}\right| \leq \max \left(\frac{\lambda}{1+\lambda},\left|\frac{1-\lambda}{1+\lambda}\right|\right)<1$ for $\lambda>0$. Hence $I \leq A_{j}(|t|) e^{-c t}$ for an appropriate polynomial $A_{j}(\cdot)$ such that

$$
\frac{1}{2}\left\|Q_{j}\right\|_{\infty} \sum_{n=0}^{j+t} \pi_{n}\left\|Q_{n}\right\|_{\infty} \int_{(-1,0)}(1-x)^{\alpha}(1+x)^{\beta} d x \leq A_{j}(|t|),
$$

and $c=-\log \left\{\max \left(\frac{\lambda}{1+\lambda},\left|\frac{1-\lambda}{1+\lambda}\right|\right)\right\}$. Such a polynomial $A_{j}$ exists since $\left\|Q_{n}\right\|_{\infty}$ grows polynomially in $n$ and $\pi_{n}$ is bounded. See formula 22.14.1 in Abramowitz and Stegun [1].

Thus $I$ is clearly bounded by the right-hand side of (3.1).

For the second term, $I I \leq \frac{1}{2} \sum_{n=0}^{j+t} \pi_{n}\left\|Q_{n} Q_{j}\right\|_{\infty} \int_{0}^{1}\left(\frac{x+\lambda}{1+\lambda}\right)^{t}(1-x)^{\alpha}(1+x)^{\beta} d x$. There we make the change of variables $s=-\log \left(\frac{x+\lambda}{1+\lambda}\right)$, and for simplicity let $x(s)=(1+\lambda) e^{-s}-\lambda$. Then the integral reduces to

$$
(1+\lambda)^{1+\alpha} \int_{0}^{\log \left(\frac{1+\lambda}{\lambda}\right)} e^{-s(t+1)}\left(1-\lambda+(1+\lambda) e^{-s}\right)^{\beta}\left(1-e^{-s}\right)^{\alpha} d s .
$$

Using the fact that $\left(1-e^{-s}\right)^{\alpha}=s^{\alpha}(1+O(s))$ and $\left(1-\lambda+(1+\lambda) e^{-s}\right)^{\beta}=$ $2^{\beta}+O(s)$, the above integral becomes

$$
(1+\lambda)^{1+\alpha} \int_{0}^{\log \left(\frac{1+\lambda}{\lambda}\right)} e^{-s(t+1)}\left(2^{\beta} s^{\alpha}+O\left(s^{\alpha+1}\right)\right) d s,
$$

where the upper bounds $O(s)$ can be made specific. Next, applying the standard asymptotic methods of Laplace to this yields the following asymptotics:

$$
\int_{0}^{\log \left(\frac{1+\lambda}{\lambda}\right)} e^{-s(t+1)} s^{\alpha} d s \asymp \frac{\Gamma(\alpha+1)}{(t+1)^{1+\alpha}} .
$$


Thus one can obtain a large enough constant $\widetilde{C}_{\alpha, \beta, \lambda}$ such that

$$
I I \leq \frac{\widetilde{C}_{\alpha, \beta, \lambda}\left\|Q_{j}\right\|_{\infty}}{(t+1)^{1+\alpha}} \sum_{n=0}^{t+j} \pi_{n}\left\|Q_{n}\right\|_{\infty} .
$$

In order to derive effective bounds on $\left\|\nu-\mu_{t}\right\|_{T V}$ it is necessary to gain a more detailed understanding of $\pi_{n}$ and $\left\|Q_{n}\right\|_{\infty}$. When $\min (\alpha, \beta) \geq-\frac{1}{2}$, the $\left\|Q_{n}\right\|_{\infty}$ can be estimated using the known maximum for the Jacobi polynomials found in Lemma 4.2 .1 on page 85 of 2 together with Koornwinder's definition of these polynomials.

One way to derive estimates for $\pi_{n}$ is to use the expression $\pi_{n}$ in terms of $p_{n}$, $r_{n}$, and $q_{n}$. For Koorwinder's class of polynomials these expressions are derived for all $\alpha, \beta, M, N$ in [4]. It can be verified directly that in the case when $M=0$, then $p_{0}=\frac{2(\alpha+1)}{(1+N)(\alpha+\beta+2)}>0$. After taking into account the normalization $Q_{n}(1)=1$, and taking into account a small typo, it can be verified from equations (41)-(45) in [4] that $p_{n}$ and $q_{n}$ are positive for $n \geq 1$. Thus the conditions for Lemma 1 are satisfied for all $\alpha, \beta>-1$. Furthermore, from (18), (19) and (32) in [4] it can be easily seen that $p_{n} \rightarrow \frac{1}{2}$ and $q_{n} \rightarrow \frac{1}{2}$ as $n \rightarrow \infty$, and hence $r_{n}=1-p_{n}-q_{n} \rightarrow 0$ as $n \rightarrow \infty$. Thus for $\lambda$ large enough, the operator $P^{\lambda}$ corresponds to a Markov chain.

As the expressions for these quantities are laborious to write down, we instead focus our attention on a specific case in which our calculations are easy to follow. Specifically we focus on the Chebyshev polynomials.

\section{Chebyshev polynomials: Upper And lower Bounds}

By applying Koorwinder's results to the Chebyshev polynomials of the first kind which correspond to the case of $\alpha=\beta=-\frac{1}{2}$, we arrive at a family of orthogonal polynomials with respect to the measure $\frac{1}{1+N}\left(\frac{1}{\pi \sqrt{1-x^{2}}} d x+N \delta_{1}(x)\right)$. Using (2.1) we find that

$$
Q_{n}(x):=-N(x+1) U_{n-1}(x)+(1+2 n N) T_{n}(x),
$$

where $T_{n}$ and $U_{n}$ denote the Chebyshev polynomials of the first and second kind respectively. Notice that $U_{n}(1)=n+1$ and $T_{n}(1)=1$, which immediately verifies that $Q_{n}(1)=1$.

Once again we consider the operator

$$
H=\left(\begin{array}{cccccc}
r_{0} & p_{0} & 0 & 0 & 0 & \ldots \\
q_{1} & r_{1} & p_{1} & 0 & 0 & \vdots \\
0 & q_{2} & r_{2} & p_{2} & 0 & \ddots \\
0 & 0 & q_{3} & r_{3} & \ddots & \ddots \\
\vdots & \ldots & \ddots & \ddots & \ddots & \ldots
\end{array}\right)
$$

on $\ell^{2}(\pi)$, so that the vector $\left(Q_{0}(x), Q_{1}(x), Q_{2}(x), \ldots\right)^{T}$ is an eigenvector with eigenvalue $x$.

Specifically the numbers $p_{n}, r_{n}$, and $q_{n}$ satisfy $p_{0} P_{1}(x)+r_{0} P_{0}(x)=x$ for $n=0$, and

$$
p_{n} Q_{n+1}(x)+r_{n} Q_{n}(x)+q_{n} Q_{n-1}(x)=x Q_{n}(x) \quad \text { for } n \geq 1 \text {. }
$$


Keisel and Wimp [4] give expressions for $p_{n}, r_{n}$ and $q_{n}$ for $n \geq 0$. To find the expressions directly in this case one could use (4.1) to derive three linearly independent equations and then solve for $p_{n}, r_{n}$, and $q_{n}$.

For the case $n=0$ the equation immediately gives us that $p_{0}=\frac{1}{N+1}$ and $r_{0}=\frac{N}{N+1}$. Evaluating at convenient choices of $x$, such as $-1,0,1$, does not yield linearly independent equations for all $n$. One solution to this is to evaluate at $x=1,-1$ and differentiate (4.1) and then evaluate at $x=0$. This gives three linearly independent equations, and a direct calculation then shows that

$$
\begin{gathered}
p_{n}=\frac{1}{2} \cdot \frac{1+(2 n-1) N}{1+(2 n+1) N}, \quad q_{n}=\frac{1}{2} \cdot \frac{1+(2 n+1) N}{1+(2 n-1) N}, \quad \text { and } \\
r_{n}=\frac{-2 N^{2}}{(1+(2 n-1) N)(1+(2 n+1) N)} .
\end{gathered}
$$

As $r_{n} \leq 0$, the operator $H$ fails to correspond to a Markov chain. However, this is the case we addressed at the end of Section 2 . Thus consider $P_{\lambda}=\frac{1}{1+\lambda}(H+$ $\lambda I)$. Now, $\left|r_{n}\right|$ is a decreasing sequence for $n \geq 1$. So provided that $\lambda \geq\left|r_{1}\right|=$ $\frac{2 N^{2}}{(1+N)(1+3 N)}$, we then have $p_{n}^{\lambda}, r_{n}^{\lambda}, q_{n}^{\lambda} \geq 0$. Thus we can consider these coefficients $p_{n}^{\lambda}, r_{n}^{\lambda}$, and $q_{n}^{\lambda}$ as the transition probabilities in a nearest neighbor random walk.

Recall that $\pi_{n}^{\lambda}=\pi_{n}=\frac{p_{0} \cdots p_{n-1}}{q_{1} \cdots q_{n}}$. Thus for $P_{\lambda}$ we can directly calculate $\pi_{n}$ from (4.2). We have that $p_{0} \cdots p_{n-1}=\frac{1}{2^{n-1}} \frac{N}{1+(2 n-1) N}$ and similarly that $q_{1} \cdots q_{n}=$ $\frac{1}{2^{n}} \frac{1+(2 n+1) N}{1+N}$. Thus $\pi_{n}=\frac{2(1+N) N}{(1+(2 n-1) N)(1+(2 n+1) N)}$.

Theorem 2. Given $N>0$ and $\lambda \geq \frac{2 N^{2}}{(1+N)(1+3 N)}$, consider the case of the Chebyshev type random walks over $\Omega=\{0,1,2, \ldots\}$ with probability operator

$$
P_{\lambda}=\left(\begin{array}{cccccc}
r_{0}^{\lambda} & p_{0}^{\lambda} & 0 & 0 & 0 & \ldots \\
q_{1}^{\lambda} & r_{1}^{\lambda} & p_{1}^{\lambda} & 0 & 0 & \vdots \\
0 & q_{2}^{\lambda} & r_{2}^{\lambda} & p_{2}^{\lambda} & 0 & \ddots \\
0 & 0 & q_{3}^{\lambda} & r_{3}^{\lambda} & \ddots & \ddots \\
\vdots & \ldots & \ddots & \ddots & \ddots & \ldots
\end{array}\right)
$$

where $p_{n}^{\lambda}=\frac{1}{2(1+\lambda)} \cdot \frac{1+(2 n-1) N}{1+(2 n+1) N}, \quad q_{n}^{\lambda}=\frac{1}{2(1+\lambda)} \cdot \frac{1+(2 n+1) N}{1+(2 n-1) N}$ and $\quad r_{n}^{\lambda}=1-p_{n}^{\lambda}-q_{n}^{\lambda}$ for $n \geq 1$, with $p_{0}^{\lambda}=\frac{1}{(1+\lambda)(N+1)}=1-r_{0}^{\lambda}$. Then for the random walk originating at some site $j \in \Omega$, there are positive constants $c$ and $C$ that depend on $j, N$ and $\lambda$ such that

$$
\frac{c}{\sqrt{t}} \leq\left\|\nu-\mu_{t}\right\|_{T V} \leq C \frac{\log t}{\sqrt{t}}
$$

for $t$ sufficiently large.

Proof. For the upper bound we simply need to estimate the sums appearing in Lemma 1. Since $\pi_{n}=O\left(\frac{1}{(n+1)^{2}}\right)$, it is easy to see that the second sum $\sum_{n=j+t+1}^{\infty} \pi_{n}$ is bounded by $C_{N} /(t+j+1)$. The main term turns out to be the first sum. 
In the case of the Chebyshev type polynomials we have the bound $\left\|Q_{n}\right\|_{\infty} \leq$ $4 N n+1$. Thus the first sum in Lemma 1 is bounded by $\hat{C}_{\alpha, \beta, \lambda, N} \frac{j \log (t+j+2)}{\sqrt{t}}$ for an appropriate constant $\hat{C}_{\alpha, \beta, \lambda, N}$, and so, for an appropriate $C$ and large $t$,

$$
\left\|\nu-\mu_{n}\right\|_{T V} \leq C \frac{\log t}{\sqrt{t}}
$$

On the other hand, recalling that $Q_{0}(x)=\pi_{0}=1$, we have that

$$
\left\|\nu-\mu_{n}\right\|_{T V} \geq\left|\int_{(-1,1)}\left(\frac{x+\lambda}{1+\lambda}\right)^{t} Q_{j}(x)\left(1+x^{\beta}\right)(1-x)^{\alpha} d x\right| .
$$

However, we have already shown that for large enough $t$, the above right-hand side is asymptotic to $\frac{\tilde{C}}{\sqrt{1+t}}$.

We finish with some concluding remarks. At first the bound $\left\|Q_{n}\right\|_{\infty} \leq 4 N n+1$ may appear somewhat imprecise, since near $x=1$ we have that $Q_{n}(1)=1$. It is tempting to suggest that the correct asymptotic for the total variation norm is $C / \sqrt{t}$. However, on closer examination in the neighborhood of $x=1, Q_{n}^{\prime}(x) \approx n^{3}$. This $n^{3}$ causes the errors to be at least of the order of the main term. Overall it seems unlikely to the authors that $C / \sqrt{t}$ is the correct asymptotic for the Chebyshev type polynomials.

\section{Comparison to Other Methods}

An ergodic Markov chain $P=(p(i, j))_{i, j \in \Omega}$ with stationary distribution $\nu$ is said to be geometrically ergodic if and only if there exist $0<R<1$ and a function $M: \Omega \rightarrow \mathbb{R}_{+}$such that for each initial state $i \in \Omega$, the total variation distance decreases exponentially as follows:

$$
\left\|p_{t}(i, \cdot)-\nu(\cdot)\right\|_{T V}=\frac{1}{2} \sum_{j \in \Omega}\left|p_{t}(i, j)-\nu(j)\right| \leq M(i) R^{t} .
$$

In other words, an ergodic Markov chain is geometric when the rate of convergence to stationary distribution is exponential. See [8] and the references therein.

If the state space $\Omega$ is finite, $|\Omega|=d<\infty$, and the Markov chain is irreducible and aperiodic, then $P$ will have eigenvalues that can be ordered as follows:

$$
\lambda_{1}=1>\left|\lambda_{2}\right| \geq \cdots \geq\left|\lambda_{d}\right| .
$$

In this case, the Perron-Frobenious Theorem will imply geometric ergodicity with

$$
\left\|p_{t}(i, \cdot)-\nu(\cdot)\right\|_{T V}=O\left(t^{m_{2}-1}\left|\lambda_{2}\right|^{t}\right),
$$

where $m_{2}$ is the algebraic multiplicity of $\lambda_{2}$. Here the existence of a positive spectral gap, $1-\left|\lambda_{2}\right|>0$, implies geometric ergodicity with the exponent $-\log \left|\lambda_{2}\right| \approx 1-\left|\lambda_{2}\right|$ whenever the spectral gap is small enough.

When dealing with Markov chains over general countably infinite state space $\Omega$, the existence of a positive spectral gap of the operator $P$ is essentially equivalent to the chain being geometrically ergodic. For instance, the orthogonal polynomial approach in [7] resulted in establishing the geometric rate $R=\max \left\{r+2 \sqrt{p q}, \frac{q}{q+r}\right\}$ 
for the Markov chain

$$
P=\left(\begin{array}{ccccc}
0 & 1 & 0 & 0 & \ldots \\
q & r & p & 0 & \cdots \\
0 & q & r & p & \ddots \\
0 & 0 & q & r & \ddots \\
\vdots & \vdots & \ddots & \ddots & \ddots
\end{array}\right) \quad q>p, r>0
$$

over $\Omega=\mathbb{Z}_{+}$, together with establishing the value of the spectral gap, $1-R>0$.

As for the Markov chain $P_{\lambda}$ considered in Theorem 2 of this paper, its spectral measure $\frac{1}{1+\lambda} d \psi((1+\lambda) x-\lambda)$ over $\left(\frac{\lambda-1}{\lambda+1}, 1\right]$ admits no spectral gap between the point mass at 1 and the rest of the spectrum implying sub-geometric ergodicity. The sub-exponential rate in total variation norm is then estimated to be of polynomial order between $\frac{1}{\sqrt{t}}$ and $\frac{\log t}{\sqrt{t}}$.

In the field of probability and stochastic processes, there is great interest in finding methods for analyzing Markov chains over general state space that have polynomial rates of convergence to stationary distribution. In Menshikov and Popov [9] a one dimensional version of Lamperti's problem is considered. There, a class of ergodic Markov chains on countably infinite state space with sub-exponential convergence to the stationary probabilities is studied via probabilistic techniques. One of their results relates to our main result, Theorem 2. Namely, Theorem 3.1 of [9, when applied to our case, implies for any $\varepsilon>0$ the existence of positive real constants $C_{1}$ and $C_{2}$ such that

$$
C_{1} t^{-\frac{1}{2}-\varepsilon} \leq\left|\nu(0)-\mu_{t}(0)\right| \leq C_{2} t^{-\frac{1}{2}+\varepsilon} .
$$

Thus for the Markov chain considered in Theorem 2, the orthogonal polynomial approach provides a closed form expression for the difference $\nu-\mu_{t}$, and a significantly sharper estimate on the convergence of $\mu_{t}$ to the stationary distribution $\nu$, for both the single state distance $\left|\nu(0)-\mu_{t}(0)\right|$ and a much stronger total variation norm, $\left\|\nu-\mu_{t}\right\|_{T V}$.

\section{ACKNOWLEDGMEnTs}

The authors would like to thank Yuan Xu of the University of Oregon for his helpful comments which initiated this work. They would also like to thank Michael Anshelevich of Texas A\&M for the feedback he provided during the conference on orthogonal polynomials in probability theory in July of 2010. They would like to thank Andrew R. Wade of the University of Strathclyde for his helpful comments on the preprint of this paper. Finally, they would like to thank the anonymous referee for the many helpful corrections and suggestions.

\section{REFERENCES}

[1] Handbook of mathematical functions with formulas, graphs, and mathematical tables, Dover Publications Inc., New York, 1992. Edited by Milton Abramowitz and Irene A. Stegun; Reprint of the 1972 edition. MR 1225604 (94b:00012)

[2] Mourad E. H. Ismail, Classical and quantum orthogonal polynomials in one variable, Encyclopedia of Mathematics and its Applications, vol. 98, Cambridge University Press, Cambridge, 2005. With two chapters by Walter Van Assche; With a foreword by Richard A. Askey. MR2191786(2007f:33001) 
[3] Samuel Karlin and James McGregor, Random walks, Illinois J. Math. 3 (1959), 66-81. MR0100927 (20 \#7352)

[4] Harry Kiesel and Jet Wimp, A note on Koornwinder's polynomials with weight function $(1-x)^{\alpha}(1+x)^{\beta}+M \delta(x+1)+N \delta(x-1)$, Numer. Algorithms 11 (1996), no. 1-4, 229-241, DOI 10.1007/BF02142499. Orthogonal polynomials and numerical analysis (Luminy, 1994). MR.1383387 (97a:33020)

[5] Tom H. Koornwinder, Orthogonal polynomials with weight function $(1-x)^{\alpha}(1+x)^{\beta}$ $+M \delta(x+1)+N \delta(x-1)$, Canad. Math. Bull. 27 (1984), no. 2, 205-214, DOI 10.4153/ CMB-1984-030-7. MR740416 (85i:33011)

[6] Yevgeniy Kovchegov, Orthogonality and probability: beyond nearest neighbor transitions, Electron. Commun. Probab. 14 (2009), 90-103, DOI 10.1214/ECP.v14-1447. MR2481669 (2010c:60113)

[7] Yevgeniy Kovchegov, Orthogonality and probability: mixing times, Electron. Commun. Probab. 15 (2010), 59-67, DOI 10.1214/ECP.v15-1525. MR2595683(2011c:60237)

[8] Sean Meyn and Richard L. Tweedie, Markov chains and stochastic stability, 2nd ed., Cambridge University Press, Cambridge, 2009. With a prologue by Peter W. Glynn. MR2509253 (2010h:60206)

[9] M. V. Menshikov and S. Yu. Popov, Exact power estimates for countable Markov chains, Markov Process. Related Fields 1 (1995), no. 1, 57-78. MR1403077 (98f:60132)

[10] G. Szegö, Orthogonal Polynomials, Fourth ed., Vol. 23, AMS Colloquium Publications, 1975. MR.0372517 (51:8724)

[11] V. B. Uvarov, Relation between systems of polynomials orthogonal with respect to various distribution functions, Z̆. Vychisl. Mat. i Mat. Fiz. (USSR) 9 (1969), no. 6, 1253-1262. MR.0262764 (41:7369)

Department of Mathematics, Oregon State University, Corvallis, Oregon 97331

E-mail address: kovchegy@math.oregonstate.edu

Department of Mathematics, Oregon State University, Corvallis, Oregon 97331

E-mail address: Nicholas.Michalowski@math.oregonstate.edu 